\title{
THE NATURE OF THE ATMOSPHERE OF THE TRANSITING SUPER-EARTH GJ 1214b
}

\author{
Eliza Miller-Ricci and Jonathan J. Fortney \\ Department of Astronomy and Astrophysics, University of California, Santa Cruz, CA 95064, USA; elizamr@ucolick.org \\ Received 2010 January 6; accepted 2010 April 26; published 2010 May 24
}

\begin{abstract}
The newly discovered planet GJ $1214 \mathrm{~b}$ is the first known transiting super-Earth requiring a significant atmosphere to explain its observed mass and radius. Models for the structure of this planet predict that it likely possesses an $\mathrm{H}-\mathrm{He}$ envelope of at least $0.05 \%$ of the total mass of the planet. However, models without a significant $\mathrm{H}-\mathrm{He}$ atmosphere are not entirely ruled out by the available data. Here, we explore a range of possible atmospheres for the planet, ranging from solar composition gas, to pure $\mathrm{CO}_{2}$ or water (steam). We present transmission and emission spectra for each of these cases. We find that, if GJ 1214b possesses a hydrogenrich atmosphere as expected, then the primary transit depth for such an atmosphere would vary at a level of up to $0.3 \%$ as a function of wavelength, relative to the background light of its M-dwarf host star. Observations at this level of precision are potentially obtainable with current space-based instrumentation. Successful detection of the transmission signature of this planet at the $\sim 0.1 \%$ level would therefore provide confirmation of the hydrogen-rich nature of the planetary atmosphere. It follows that transmission spectroscopy at this level of precision could provide a first glimpse into answering the question of whether planets in the super-Earth mass regime $\left(1-10 M_{\oplus}\right)$ more closely resemble large terrestrial planets or small gas giant planets.
\end{abstract}

Key words: planetary systems

Online-only material: color figures

\section{INTRODUCTION}

The newly discovered class of super-Earths (planets with masses between 1 and $10 M_{\oplus}$ ) brings planet detection into a mass regime that has not previously been studied, due to the lack of such planets in our solar system. The recent detection of the first transiting super-Earths (Léger et al. 2009; Charbonneau et al. 2009) has allowed for the first measurements of mass and radius, and therefore bulk density of these objects. Since superEarths lie in an intermediate mass range between terrestrial planets and the gas/ice giants of our solar system, the question arises as to where the dividing line lies between these two classes of planets. Super-Earths with thick hydrogen/helium atmospheres ("sub-Neptunes") may have experienced formation histories that are similar to those of other gas giants, whereas predominantly solid planets that are true scaled up analogs of the terrestrial planets in our solar system would instead be thought of as "super" or large Earths.

GJ 1214b (Charbonneau et al. 2009) is the second known transiting super-Earth, and also the first such planet to likely possess significant gaseous envelope. The planet's mass $\left(6.6 M_{\oplus}\right)$ and radius $\left(2.7 R_{\oplus}\right)$ imply a density that is too low to be explained by rock and water ice alone. Indeed, a comparison against theoretical mass-radius relationships predicted for solid exoplanets (Valencia et al. 2006; Fortney et al. 2007; Seager et al. 2007; Sotin et al. 2007) reveals that GJ 1214 b lies in a region of parameter space where a significant $\mathrm{H} / \mathrm{He}$ atmosphere is the most likely explanation for the planet's observed properties. In their discovery paper, Charbonneau et al. (2009) state that a lowdensity composition for the planet, made up of $75 \%$ water (ice), $22 \%$ silicate rock, and $3 \%$ iron would still necessitate an $\mathrm{H} / \mathrm{He}$ atmosphere of 0.05 mass percent to explain the planet's radius. This corresponds to an $\mathrm{H} / \mathrm{He}$ atmosphere of approximately 70 bars. Such a planet lies somewhere on the spectrum between terrestrial planets, and gas or ice giants, making this a highly interesting new object for study.
The presence of a significant H/He atmosphere on GJ $1214 \mathrm{~b}$ is not confirmed, however, by the mass and radius measurements for this planet. Rogers \& Seager (2009) find that the observed mass and radius of GJ $1214 \mathrm{~b}$ can be explained if the planet is composed of at least $47 \%$ water accompanied by a massive steam atmosphere. Additionally, Charbonneau et al. (2009) point out that their measured radius of the planet's M-dwarf host star, GJ 1214, is inconsistent with theoretical models. If, in fact, the radius of the star is $15 \%$ smaller, as predicted by models (Baraffe et al. 1998), then the radius of the planet, GJ 1214b, would also be smaller by a comparable amount-resulting in a planet that does not require a large atmosphere to explain its observed radius.

Given the uncertainties in the nature of the atmosphere of GJ 1214b, follow-up observations aimed at detecting the spectral signature of the planet's atmosphere will be necessary to confirm its molecular makeup. This could be accomplished via secondary eclipse (emission) or occultation (transmission) spectroscopy. Both methods have been shown to be successful in characterizing the atmospheres of gas giant exoplanets (e.g., Charbonneau et al. 2002; Deming et al. 2005; Swain et al. 2008; Knutson et al. 2008).

Miller-Ricci et al. (2009) have pointed out that transmission spectroscopy is an excellent tool for determining the hydrogen content of an exoplanet atmosphere. Since the depth of spectral features in transmission is proportional to the atmospheric scale height (which in turn scales inversely proportionally to the mean molecular weight of the planetary atmosphere), a hydrogen-rich atmosphere will exhibit spectral features in transmission that are up to a factor of 20 larger than those of a hydrogen-depleted $\mathrm{CO}_{2}$ atmosphere. For GJ $1214 \mathrm{~b}$, the expected signature of a hydrogen-rich atmosphere would manifest itself on a level of $\sim 0.1 \%$ relative to the M-dwarf (M4.5) host star. Such precision has been shown to be obtainable with current space-based observatories_-both Hubble (e.g., Swain et al. 2008; Pont et al. 2009) and Spitzer (e.g., Deming et al. 2007). In this Letter, 
we present calculations of transmission and emission spectra for possible atmospheres on the planet GJ 1214b, ranging in composition from hydrogen-rich to hydrogen-poor. We present our model and its parameters in Section 2. We then present our resulting spectra in Section 3; and we conclude with some thoughts on combining our models with observations to constrain the nature of GJ 1214b's atmosphere in Section 4.

\section{METHODOLOGY}

\subsection{Range of Model Parameters}

We consider atmospheres for GJ 1214b ranging from hydrogen-dominated solar composition gas to hydrogen- and helium-depleted atmospheres made up of predominantly heavier molecules. The six different cases of atmospheric composition that we investigate are as follows.

1. Solar composition atmosphere. For this case and the two that follow, the molecular species that make up the atmosphere appear in equilibrium abundances (see Section 2.2 for more details).

2. $30 \times$ Enhanced metallicity atmosphere. Here, we use the base composition of atmosphere no. 1 , but in this case the abundances of all species except for $\mathrm{H}$ and $\mathrm{He}$ are enhanced by a factor of 30 .

3. $50 \times$ Enhanced metallicity atmosphere. Here, the metallicity is enhanced 50 times relative to solar.

4. $100 \%$ water (steam) atmosphere.

5. $50 \%$ water, $50 \% \mathrm{CO}_{2}$ atmosphere.

6. $\mathrm{CO}_{2}$ atmosphere plus trace gasses. This model atmosphere is composed of $96.5 \% \mathrm{CO}_{2}$ with other trace gasses appearing in Venusian abundances, notably $3.5 \% \mathrm{~N}_{2}$ and $20 \mathrm{ppm}$ $\mathrm{H}_{2} \mathrm{O}$.

Simple energy balance arguments for the atmosphere of GJ $1214 \mathrm{~b}$ yield a day-side equilibrium temperature of $660 \mathrm{~K}$ for redistribution of incident energy over the day-side only, and $555 \mathrm{~K}$ for efficient redistribution over the entire planet, if the planet's Bond albedo is zero. Marley et al. (1999) found that all planets orbiting M-dwarfs should have very low albedos, in the absence of clouds or surfaces that efficiently scatter incoming starlight. The reason for this is that M-stars give off most of their light in the IR, rather than the visible, and at these wavelengths Rayleigh scattering is extremely inefficient. The planet therefore absorbs nearly all of the incident starlight, rather than scattering it away. For $\mathrm{H} / \mathrm{He}$ dominated atmospheres, we calculate self-consistent models that justify this zero-albedo assumption. To bracket the likely range of atmospheric temperatures, we compute models with day-side and planet-wide energy redistribution $-T_{\mathrm{eq}}=660$ and $555 \mathrm{~K}$, respectively.

\subsection{Description of the Model Atmosphere}

For the three hydrogen-dominated compositions that we consider (cases 1, 2, and 3), we calculate temperature-pressure $(T-P)$ profiles using the LTE model atmosphere code of Marley \& McKay (1999), as applied to irradiated exoplanets by Fortney et al. $(2005,2006,2008)$. For the remaining three hydrogendepleted atmospheres (cases 4,5 , and 6), we calculate $T-P$ profiles according to the model developed in Miller-Ricci et al. (2009). Both model atmospheres determine radiative equilibrium $T-P$ profiles in hydrostatic equilibrium, which then switch over to adiabatic profiles in regions where the atmosphere is found to be convectively unstable. The Fortney et al. model

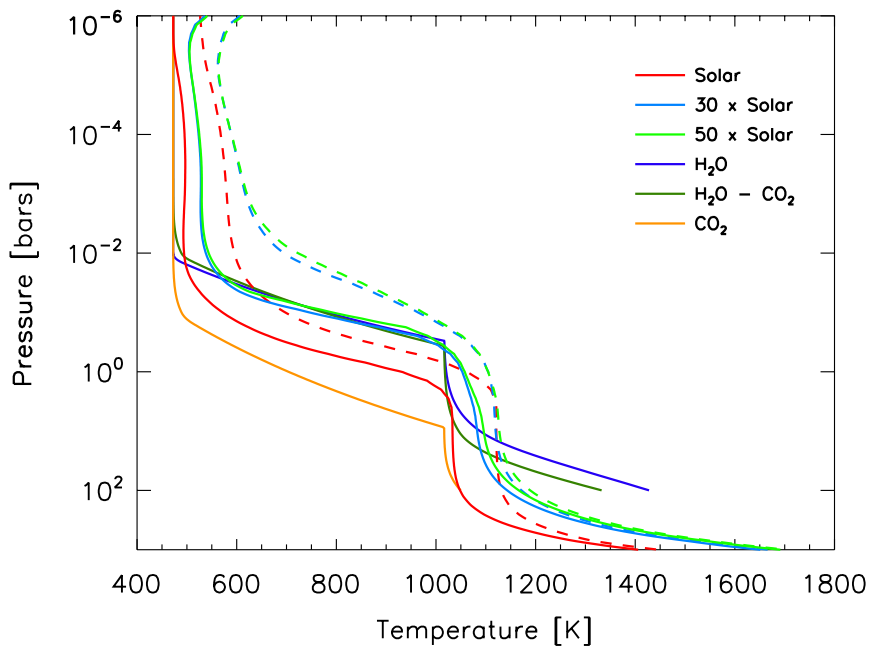

Figure 1. $T-P$ profiles for the various atmospheres presented in this Letter. Solid lines indicate models where the day- to night-side redistribution of heat is efficient $\left(T_{\mathrm{eff}}=555 \mathrm{~K}\right)$. Dashed lines indicate models where the planet reradiates all of its heat from the day side of the planet only $\left(T_{\text {eff }}=660 \mathrm{~K}\right)$.

(A color version of this figure is available in the online journal.)

self-consistently calculates the atmospheric temperature structure by determining the levels where the incident flux from the host star is absorbed into the planetary atmosphere as a function of wavelength, and balancing this energy input against the re-emitted flux from the planet. (See Freedman et al. (2008) for a description of the opacity database.) We find a Bond albedo of $0.003-0.015$ for all models, validating the approximation of a zero Bond albedo. The Miller-Ricci et al. (2009) model is simpler, in that it uses a gray approximation for the atmospheric opacities and temperature profile, following the formalism presented in Hansen (2008) for an irradiated gray atmosphere-their Equation (45). The resulting $T-P$ profiles for the six different cases of atmospheric composition that we consider are presented in Figure 1. Our model does not include cases with inverted temperature profiles or sources of non-LTE emission. Both of these effects have been implied through observations of transiting hot-Jupiters (Swain et al. 2010; Rogers et al. 2009; Machalek et al. 2009; Knutson et al. 2009, 2008). However, their causes are not sufficiently well-constrained to be self-consistently included in our model at this time.

After determining the $T-P$ profile, we calculate the planet's emitted spectrum by integrating the equation of radiative transfer through the planet's atmosphere. We assume a Planckian source function, and we include the dominant sources of molecular line opacity from 0.1 to $100 \mu \mathrm{m}$ in the IR- $-\mathrm{CH}_{4}$ (Freedman et al. 2008; Karkoschka 1994; Strong et al. 1993), $\mathrm{CO}, \mathrm{NH}_{3}$ (Freedman et al. 2008, and references therein), $\mathrm{CO}_{2}$ (Rothman et al. 2005), and $\mathrm{H}_{2} \mathrm{O}$ (Freedman et al. 2008; Partridge \& Schwenke 1997). For the line profiles, we employ a Voigt broadening scheme. In addition to the line opacities, we also include collision-induced absorption (CIA) from $\mathrm{H}_{2}$ with itself, $\mathrm{He}$, and $\mathrm{CH}_{4}$ (Borysow 2002; Borysow et al. 2000, and references therein), and $\mathrm{CO}_{2}$ with $\mathrm{CO}_{2}$ interpolated between the databases of Brodbeck et al. (1991) and Gruszka \& Borysow (1997). We do not include atomic line opacities (such as the alkali metals), since we find that spectral lines from these species are not present at the low temperatures that are predicted in the atmosphere of GJ 1214b. Instead, we find that a combination of water features and Rayleigh scattering dominates the spectra between 0.1 and $1 \mu \mathrm{m}$. 


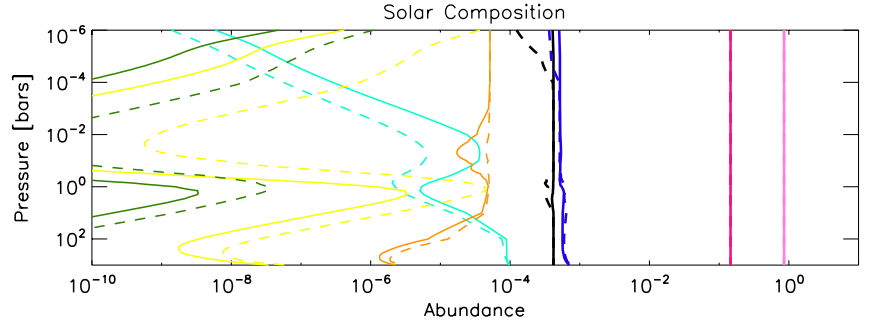

$30 \times$ Solar

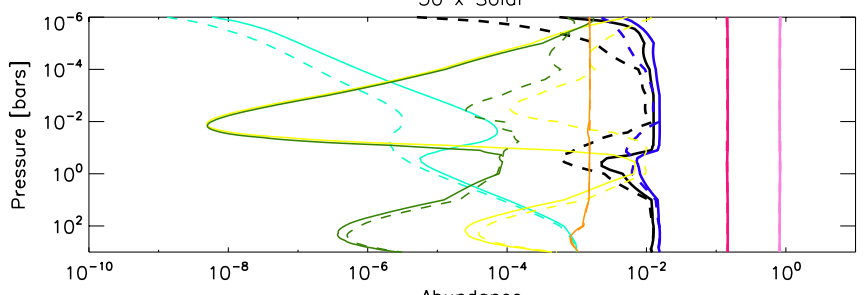

$50 \times$ Sola

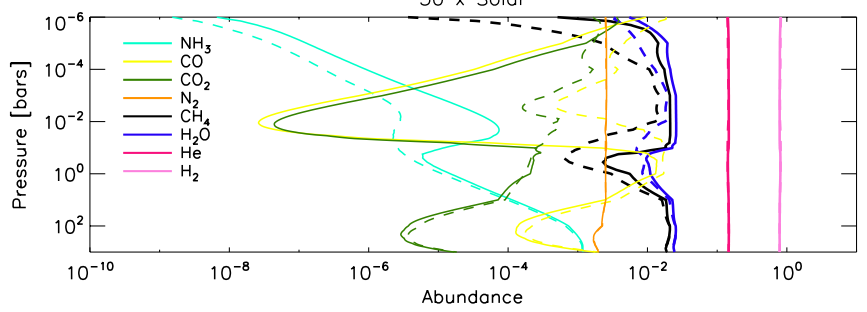

Figure 2. Equilibrium abundances as a function of atmospheric pressure for the three cases of hydrogen-dominated atmospheres. Solid and dashed lines indicate models with efficient and inefficient day-night heat redistribution, respectively. (A color version of this figure is available in the online journal.)

For calculating the transmission spectra, we use the same set of molecular line opacities as for the emission spectra; but in this case, we solve the transfer equation for a purely absorptive gas to determine the line-of-sight absorption of stellar light through the atmosphere of GJ 1214b. Here, the incident intensity from the star is calculated by interpolating the model M-dwarf spectra of Hauschildt et al. (1999) to produce a spectrum at the observed temperature $(3026 \mathrm{~K})$ and surface gravity ( $\log g=$ 4.99 in cgs units) of the host star GJ 1214 (Charbonneau et al. 2009).

For the three hydrogen-dominated atmospheres, we determine molecular abundances in chemical equilibrium, shown in Figure 2, using the code described in Miller-Ricci et al. (2009). However, the derivation of the $T-P$ profiles utilizes the equilibrium chemical abundances of Lodders \& Fegley $(2002,2006)$. Both codes produce consistent mixing ratios for a large number of molecular and atomic species.

\section{PROBING THE COMPOSITION OF GJ 1214b's ATMOSPHERE}

We present our modeled transmission spectra for GJ 1214b in Figure 3. Assuming that the transmission spectrum probes levels in the planetary atmosphere over a range of 10 scale heights $(H)$, the relative change in transit depth seen throughout the transmission spectrum is then given by

$$
\Delta D \sim \frac{20 H R_{\mathrm{pl}}}{R_{*}^{2}} .
$$

For the case of GJ 1214b, for a hydrogen-dominated atmosphere, this results in a wavelength-dependent transmission spectrum that can vary in strength by up to $0.3 \%$ (relative to the stellar light) as a function of wavelength. Despite the small size of the transiting planet, this is a large transmission signature, comparable to the transmission signals that have been successfully observed for other (much larger) hot-Jupiter systems. The reason for the large transmission signature is that GJ $1214 \mathrm{~b}$ orbits a very small $\left(\mathrm{M} 4.5, R_{*}=0.21 R_{\odot}\right)$ star, while the scale height for a hydrogen-dominated atmosphere on this planet is quite large-approximately 150-200 km.

Transmission spectra for the three hydrogen-rich atmospheres are dominated by absorption features due to water and methane. Methane is a particularly interesting species in the super-Earth
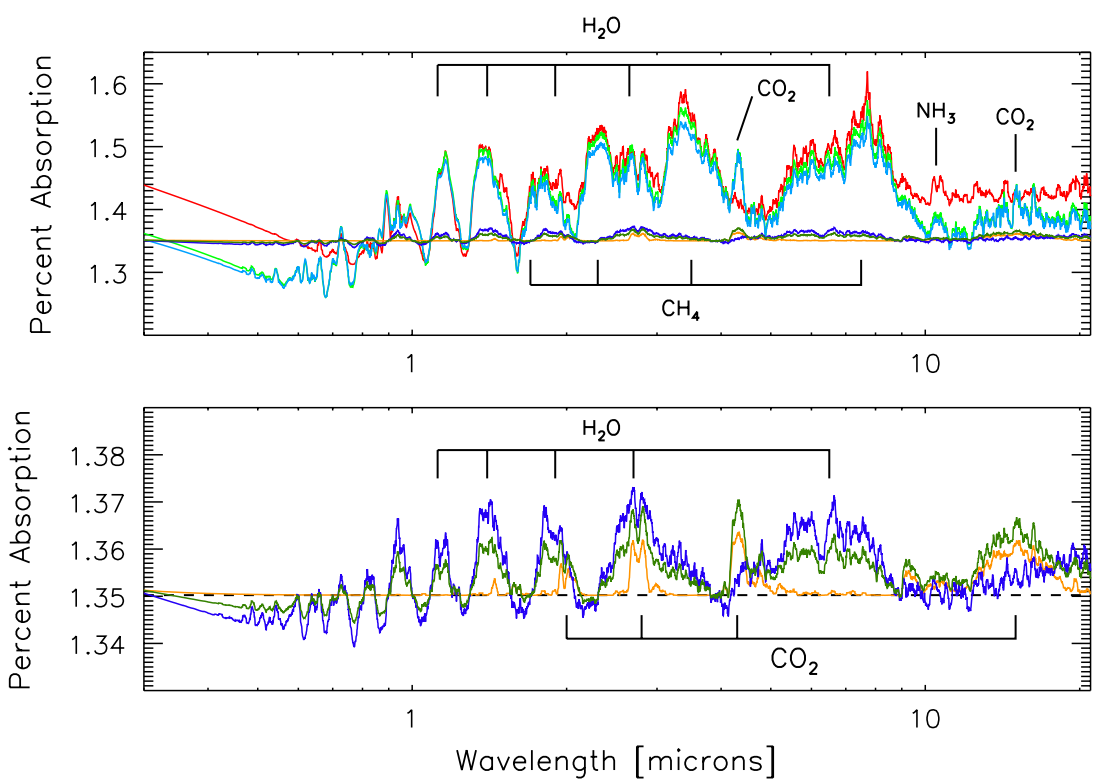

Figure 3. Top: transmission spectra for atmospheres with differing composition—solar composition (red), $30 \times$ solar metallicity (blue), $50 \times$ solar metallicity (light green), water steam (purple), $50 \%$ water-50\% $\mathrm{CO}_{2}$ (dark green), $\mathrm{CO}_{2}$ with trace quantities of water (orange). All spectra are for models with efficient day-night circulation. The spectra have been normalized to the planet's observed radius of $2.678 R_{\oplus}$ in the MEarth bandpass covering $650-1050 \mu \mathrm{m}$. Bottom: same as above but zoomed in to show the spectra for the three atmospheres composed of heavier molecules. The dashed black line indicates the radius of a planet with no atmosphere.

(A color version of this figure is available in the online journal.) 

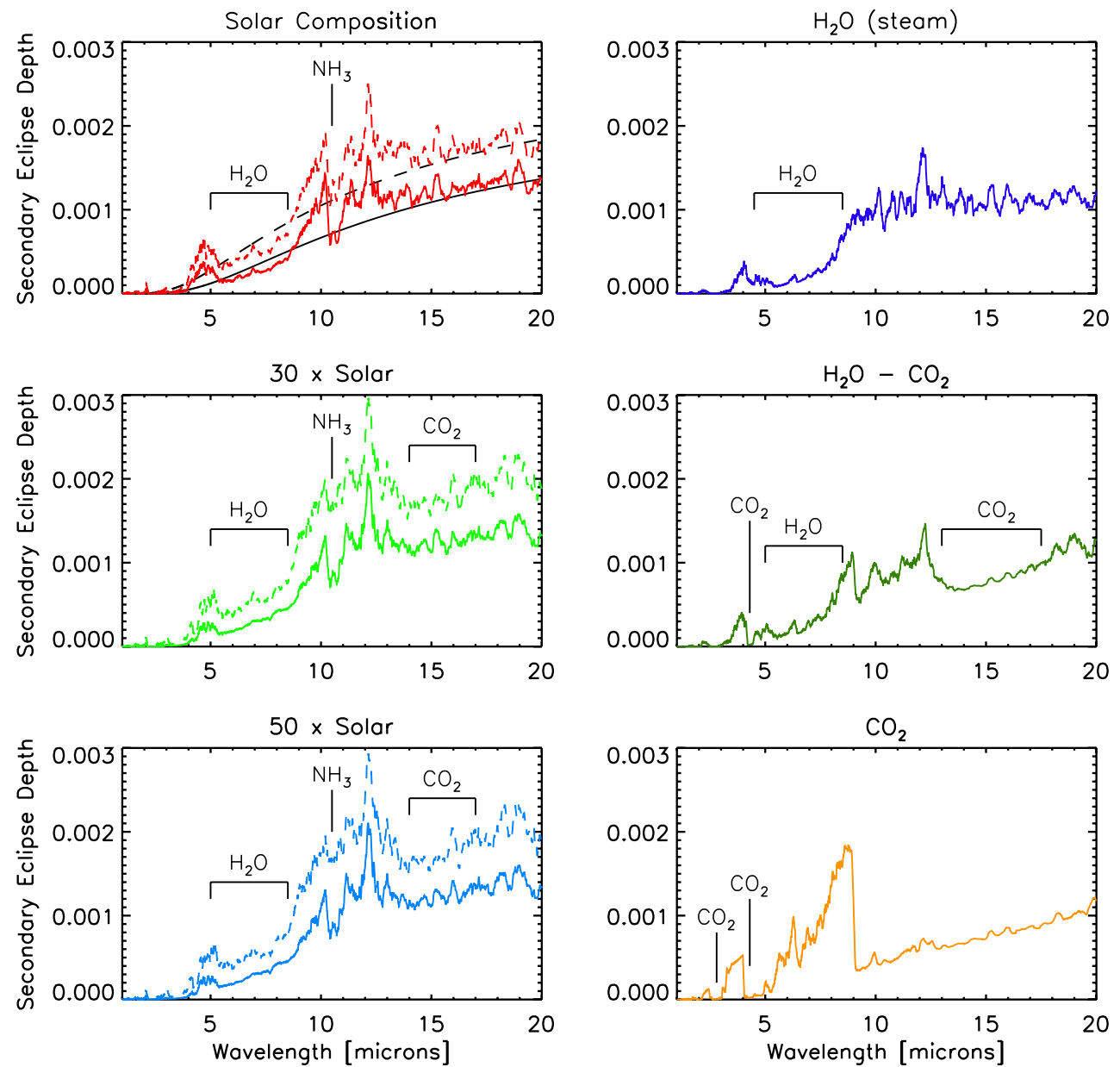

Figure 4. Contrast ratio between the day-side emission from GJ 1214b and the emitted light from its M-dwarf host star, plotted as a function of wavelength for six different possible atmospheric compositions. In the top left panel, we overplot the contrast ratios that would be expected if the planet and star both emitted as blackbodies, with planetary $T_{\text {eff }}$ of $555 \mathrm{~K}$ and $660 \mathrm{~K}$ (thin black lines). Dashed lines are spectra for models with inefficient day-night heat redistribution. Solid lines denote models with efficient heat circulation.

(A color version of this figure is available in the online journal.)

atmosphere, since photochemical reactions that destroy methane may occur faster than reactions that create this molecule (Zahnle et al. 2009a). The net result would be an observed methane abundance that is shifted away from its equilibrium value. The methane spectral features at 2.3, 3.5, and $7.5 \mu \mathrm{m}$ are therefore interesting diagnostic lines for learning about photochemistry in the atmosphere of GJ $1214 \mathrm{~b}$. Another potential consequence of methane destruction is the possibility of forming soots, which in principle could be an additional gray opacity source (Zahnle et al. 2009a) and could furthermore cause a temperature inversion, therefore altering the structure of the planetary atmosphere. Such effects must be modeled carefully, and are not included in our current calculation. Like methane, $\mathrm{NH}_{3}$ is also highly susceptible to photodissociation, and the presence (or absence) of the ammonia feature at $10.5 \mu \mathrm{m}$ is therefore another useful indicator of photochemical processes at work.

The transmission spectra from the top panel of Figure 3 additionally exhibit $\mathrm{CO}_{2}$ features in some of the hydrogendominated atmospheres. In particular, the $\mathrm{CO}_{2}$ features at 4.3 and $15 \mu \mathrm{m}$ are only present in the atmospheres with enhanced metallicity, resulting from the higher $\mathrm{C}$ and $\mathrm{O}$ abundances in these atmospheres (relative to solar). Observations at these particular wavelengths can therefore be used to probe the metal content of the super-Earth atmosphere (e.g., Zahnle et al. 2009b; Fortney et al. 2010). We caution, however, that this conclusion is dependent on our assumption of chemical equilibrium, which has been questioned by a number of observations of transiting extrasolar giant planets (Swain et al. 2009, 2008).

In contrast to the hydrogen-rich atmospheres, the three hydrogen-depleted atmospheres produce predicted transmission signatures at a level of $0.01 \%-0.02 \%$ relative to the M-star (bottom panel of Figure 3), due to the reduced scaled heights in these atmospheres. Observations at this level of precision will be very challenging, given the demonstrated capabilities of Hubble and Spitzer. If follows that observations with instruments aboard either of these telescopes would result in measurements that would be consistent with a flat spectrum. Space-based observations revealing a flat spectrum for GJ $1214 \mathrm{~b}$ would therefore rule out the presence of a cloud-free hydrogen-rich atmosphere-although $\mathrm{H} / \mathrm{He}$ atmospheres with haze or cloud layers that extend high into the atmosphere may not be excluded. However, spectra taken at a precision of at least several parts in $10^{5}$ would be necessary to discriminate between atmospheres composed of $\mathrm{H}_{2} \mathrm{O}, \mathrm{CO}_{2}$, a mix of these two gasses, or something else entirely. Detection of water features between 1 and $3 \mu \mathrm{m}$ and the $\mathrm{CO}_{2}$ features at 4.3 and $15 \mu \mathrm{m}$ may prove to be the most useful in making this distinction.

We present emission spectra for GJ 1214b in Figure 4. Hydrogen-rich atmospheres with inefficient day-night redistribution have predicted secondary eclipse depths of up to $0.3 \%$. 
This drops to approximately $0.2 \%$ if the heat redistribution is efficient. These signatures are quite large and should be readily detectable with future instrumentation such as MidInfrared Instrument aboard James Webb Space Telescope $(J W S T)$. Unfortunately, the current state of high-precision spacebased spectrophotometry is limited to the wavelength range covered by the Warm Spitzer mission. While the IRAC 3.6 and 4.5 $\mu \mathrm{m}$ filters aboard Spitzer remain in service, all longer wavelength bands, where the secondary eclipse depth is expected to be the largest, are no longer operational. Shortward of $5 \mu \mathrm{m}$, barring the presence of a thermal inversion or non-LTE emission from the planet's atmosphere, the predicted secondary eclipse depths drop below $0.1 \%$, which is likely to be prohibitively small for successful detection with Spitzer.

Longward of $5 \mu \mathrm{m}$, a whole host of water, $\mathrm{CO}_{2}$, methane, and ammonia features are predicted to be present in the secondary eclipse spectra, which could be used as diagnostics of atmospheric chemistry in the $\tau \sim 1$ region of the atmosphere. It is interesting to note however that a steam atmosphere and the $\mathrm{H} / \mathrm{He}$ dominated atmospheres actually produce quite similar emission spectra, despite their very different compositions, since water is the dominant source of opacity in both of these cases. In terms of the atmospheric structure, the emission spectra are highly dependent on the temperature structure in the planetary atmosphere and can therefore constrain the presence of temperature inversions in the upper atmosphere of GJ 1214b, as well as the total amount of day-night heat redistribution, and the possibility of non-LTE emission.

\section{DISCUSSION AND CONCLUSIONS}

Despite its observed low density, measurements of the mass and radius of GJ $1214 \mathrm{~b}$ are not sufficient to confirm the presence of a hydrogen-rich atmosphere on this planet. Instead, follow-up observations that detect the spectral signature of the planetary atmosphere will be necessary to confirm its chemical composition. Fortunately, because the planet orbits a relatively bright and nearby M-star, GJ 1214b lends itself to these types of follow-up observations. For a hydrogendominated atmosphere on GJ $1214 \mathrm{~b}$, the expected transmission signature is on the level of $0.1 \%$ relative to the host star, and the predicted emission signature (secondary eclipse depth) is also on the order of $0.1 \%$. Hydrogen-depleted atmospheres produce comparable secondary eclipse depths, but the transmission signature of an H-poor atmosphere is an order of magnitude smaller-only $0.01 \%$. Current space-based instrumentation will likely be able to detect the transmission spectrum of GJ 1214b, if the planet does indeed possess a hydrogen-rich atmosphere. However, under the assumption of no strong non-LTE emission or upper-atmosphere temperature inversions, the next generation of instruments aboard JWST will be needed to measure the emission spectrum of this planet (and its transmission spectrum if the planet is depleted in hydrogen).

GJ 1214b is currently the only known super-Earth that is accessible for space-based follow-up to determine the planet's atmospheric composition. The only other known transiting super-Earth at this time, CoRoT-7b (Léger et al. 2009), is a smaller planet orbiting a larger and hotter star. Even if this planet did have a significant atmosphere, its signature would be too small to detect against the background of its host star. The same fate will befall the super-Earths discovered by the Kepler mission (Borucki et al. 2004; Basri et al. 2005). Since most of the stars that Kepler is targeting are distant (and therefore faint) solar-type stars, the prospects for further characterization of the planets detected around these stars are not strong. One way to improve the chances of finding transiting super-Earths that are ideal for follow-up is by searching for planets around the brightest stars in the sky, such as has been proposed for all-sky missions like the Transiting Exoplanet Survey Satellite (TESS; Ricker et al. 2009). The other method is to search for transiting planets orbiting the smallest stars using networks of groundbased telescopes such as MEarth (Nutzman \& Charbonneau 2008), which is exactly how GJ 1214 b was discovered.

GJ 1214b may indeed represent a new class of planets-those which fall in the intermediate range between gas or ice giants, and terrestrial planets. As more transiting super-Earths are discovered, and the mass-radius diagram begins to fill in for these smaller planets, it will become clearer whether there is a sharp transition or a smooth continuum between the two types of planets. For now, GJ 1214b remains an interesting and unique object that is ideally suited for the type of follow-up observations that will reveal more information about the nature of this planet—-both its interior and its atmosphere.

This work was performed in part under contract with the California Institute of Technology funded by NASA through the Sagan Fellowship Program. J.J.F. thanks the Spitzer Theory Program.

\section{REFERENCES}

Baraffe, I., Chabrier, G., Allard, F., \& Hauschildt, P. H. 1998, A\&A, 337, 403 Basri, G., Borucki, W. J., \& Koch, D. 2005, New Astron. Rev., 49, 478

Borucki, W., et al. 2004, in Stellar Structure and Habitable Planet Finding, ed. F. Favata, S. Aigrain, \& A. Wilson (ESA SP-538; Noordwijk: ESA), 177

Borysow, A. 2002, A\&A, 390, 779

Borysow, A., Borysow, J., \& Fu, Y. 2000, Icarus, 145, 601

Brodbeck, C., Nguyen, V.-T., Bouanich, J.-P., Boulet, C., Jean-Louis, A., Bezard, B., \& de Bergh, C. 1991, J. Geophys. Res., 96, 17497

Charbonneau, D., Brown, T. M., Noyes, R. W., \& Gilliland, R. L. 2002, ApJ, 568,377

Charbonneau, D., et al. 2009, Nature, 462, 891

Deming, D., Harrington, J., Laughlin, G., Seager, S., Navarro, S. B., Bowman, W. C., \& Horning, K. 2007, ApJ, 667, L199

Deming, D., Seager, S., Richardson, L. J., \& Harrington, J. 2005, Nature, 434, 740

Fortney, J. J., Lodders, K., Marley, M. S., \& Freedman, R. S. 2008, ApJ, 678, 1419

Fortney, J. J., Marley, M. S., \& Barnes, J. W. 2007, ApJ, 659, 1661

Fortney, J. J., Marley, M. S., Lodders, K., Saumon, D., \& Freedman, R. 2005, ApJ, 627, L69

Fortney, J. J., Saumon, D., Marley, M. S., Lodders, K., \& Freedman, R. S. 2006, ApJ, 642, 495

Fortney, J. J., Shabram, M., Showman, A. P., Lian, Y., Freedman, R. S., Marley, M. S., \& Lewis, N. K. 2010, ApJ, 709, 1396

Freedman, R. S., Marley, M. S., \& Lodders, K. 2008, ApJS, 174, 504

Gruszka, M., \& Borysow, A. 1997, in AIP Conf. Ser. 386, The 13th International Conference of Spectral Line Shapes, ed. M. Zoppi \& L. Ulivi (Melville, NY: AIP), 429

Hansen, B. M. S. 2008, ApJS, 179, 484

Hauschildt, P. H., Allard, F., \& Baron, E. 1999, ApJ, 512, 377

Karkoschka, E. 1994, Icarus, 111, 174

Knutson, H. A., Charbonneau, D., Allen, L. E., Burrows, A., \& Megeath, S. T. 2008, ApJ, 673, 526

Knutson, H. A., Charbonneau, D., Burrows, A., O’Donovan, F. T., \& Mandushev, G. 2009, ApJ, 691, 866

Léger, A., et al. 2009, A\&A, 506, 287

Lodders, K., \& Fegley, B. 2002, Icarus, 155, 393

Lodders, K., \& Fegley, B., Jr. 2006, Chemistry of Low Mass Substellar Objects (Berlin: Springer)

Machalek, P., McCullough, P. R., Burrows, A., Burke, C. J., Hora, J. L., \& Johns-Krull, C. M. 2009, ApJ, 701, 514

Marley, M. S., Gelino, C., Stephens, D., Lunine, J. I., \& Freedman, R. 1999, ApJ, 513,879

Marley, M. S., \& McKay, C. P. 1999, Icarus, 138, 268

Miller-Ricci, E., Seager, S., \& Sasselov, D. 2009, ApJ, 690, 1056 
Nutzman, P., \& Charbonneau, D. 2008, PASP, 120, 317

Partridge, H., \& Schwenke, D. W. 1997, J. Chem. Phys., 106, 4618

Pont, F., Gilliland, R. L., Knutson, H., Holman, M., \& Charbonneau, D. 2009, MNRAS, 393, L6

Ricker, G. R., et al. 2009, BAAS, 41, 193

Rogers, J. C., Apai, D., López-Morales, M., Sing, D. K., \& Burrows, A. 2009, ApJ, 707, 1707

Rogers, L. A., \& Seager, S. 2009, arXiv:0912.3243

Rothman, L. S., et al. 2005, J. Quant. Spectrosc. Radiat. Transfer, 96, 139

Seager, S., Kuchner, M., Hier-Majumder, C., \& Militzer, B. 2007, ApJ, 669, 1279
Sotin, C., Grasset, O., \& Mocquet, A. 2007, Icarus, 191, 337

Strong, K., Taylor, F. W., Calcutt, S. B., Remedios, J. J., \& Ballard, J. 1993, J. Quant. Spectrosc. Radiat. Transfer, 50, 363

Swain, M. R., Vasisht, G., \& Tinetti, G. 2008, Nature, 452, 329

Swain, M. R., Vasisht, G., Tinetti, G., Bouwman, J., Chen, P., Yung, Y., Deming, D., \& Deroo, P. 2009, ApJ, 690, L114

Swain, M. R., et al. 2010, Nature, 463, 637

Valencia, D., O'Connell, R. J., \& Sasselov, D. 2006, Icarus, 181, 545

Zahnle, K., Marley, M. S., \& Fortney, J. J. 2009a, arXiv:0911.0728

Zahnle, K., Marley, M. S., Freedman, R. S., Lodders, K., \& Fortney, J. J. 2009b, ApJ, 701, L20 